\section{FPGA Implementation(s) of a Scalable Encryption Algorithm}

\author{
F. Macé*, F.-X. Standaert ${ }^{\dagger}$, J.-J. Quisquater \\ UCL Crypto Group, Laboratoire de Microélectronique, \\ Université Catholique de Louvain, \\ Place du Levant, 3, B-1348 Louvain-La-Neuve, Belgium \\ email: \{mace,fstandae,quisquater\}@uclouvain.be
}

\begin{abstract}
SEA is a scalable encryption algorithm targeted for small embedded applications. It was initially designed for software implementations in controllers, smart cards or processors. In this letter, we investigate its performances in recent FPGA devices. For this purpose, a loop architecture of the block cipher is presented. Beyond its low cost performances, a significant advantage of the proposed architecture is its full flexibility for any parameter of the scalable encryption algorithm, taking advantage of generic VHDL coding. The letter also carefully describes the implementation details allowing us to keep small area requirements. Finally, a comparative performance discussion of SEA with the Advanced Encryption Standard Rijndael and ICEBERG (a cipher purposed for efficient FPGA implementations) is proposed. It illustrates the interest of platform/context-oriented block cipher design and, as far as SEA is concerned, its low area requirements and reasonable efficiency.
\end{abstract}

\section{INTRODUCTION}

SEA is a parametric block cipher for resource constrained systems (e.g. sensor networks, RFIDs) that has been introduced in [1]. It was initially designed as a low-cost encryption/authentication routine (i.e. with small code size and memory) targeted for processors with a limited instruction set (i.e. AND, OR, XOR gates, word rotation and modular addition). Additionally and contrary to most recent block ciphers (e.g. the DES [2] and AES Rijndael [3], [4]), the algorithm takes the plaintext, key and the bus sizes as parameters and therefore can be straightforwardly adapted to various implementation contexts and/or security requirements. Compared to older solutions for low cost encryption like TEA (Tiny Encryption Algorithm) [5] or Yuval's proposal [6], SEA also benefits from a stronger security analysis, derived from recent advances in block cipher design/cryptanalysis.

In practice, SEA has been proven to be an efficient solution for embedded software applications using microcontrollers, but its hardware performances have not yet been investigated. Consequently, and as a first step towards hardware performance analysis, this letter explores the features of a low cost FPGA encryption/decryption core for SEA. In addition to the performance evaluation, we show that the algorithm's scalability can be turned into a fully generic VHDL design, so that any text, key and bus size can be straightforwardly re-implemented without any modification of the hardware description language, with standard synthesis and implementation tools.

\footnotetext{
* PhD Student funded by the FRIA Grant, Belgium.

$\dagger$ Postdoctoral researcher of the Belgian Fund for Scientific Research.
}

In the rest of the letter, we first provide a brief description of the algorithm specifications. Then we describe the details of our generic loop architecture and its implementation results. Finally, we discuss some illustrative comparisons of the hardware performances of SEA, the AES Rijndael and ICEBERG (a cipher purposed for efficient FPGA implementations) with respect to their design approach (e.g. flexible vs. platform/context-oriented).

\section{AlgORITHM DESCRIPTION}

\section{A. Parameters and definitions}

SEA $_{n, b}$ operates on various text, key and word sizes. It is based on a Feistel structure with a variable number of rounds, and is defined with respect to the following parameters:

- $n$ : plaintext size, key size.

- $b$ : processor (or word) size.

- $n_{b}=\frac{n}{2 b}$ : number of words per Feistel branch.

- $n_{r}$ : number of block cipher rounds.

As only constraint, it is required that $n$ is a multiple of $6 b$ (see [1] for the details). For example, using an 8-bit processor, we can derive a 96-bit block ciphers, denoted as $\mathrm{SEA}_{96,8}$.

Let $x$ be a $\frac{n}{2}$-bit vector. We consider two representations:

- Bit representation: $x_{b}=x\left(\frac{n}{2}-1\right) \ldots x(2) x(1) x(0)$.

- Word representation: $x_{W}=x_{n_{b}-1} x_{n_{b}-2} \ldots x_{2} x_{1} x_{0}$.

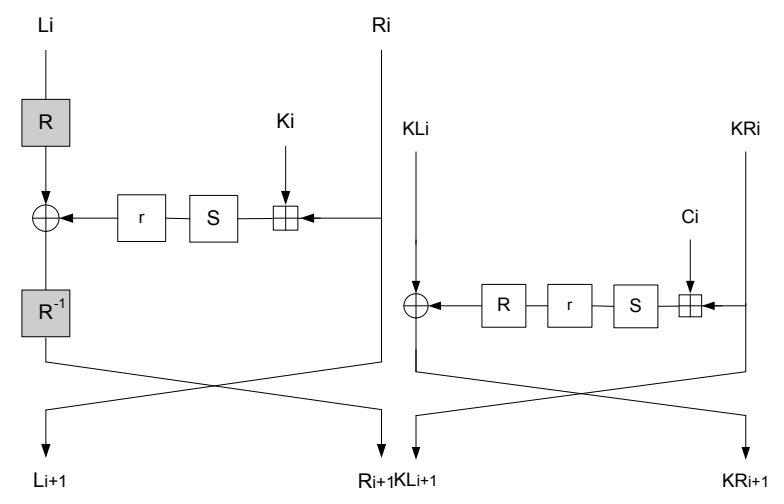

Fig. 1. Encrypt/decrypt round and key round.

\section{B. Basic operations}

Due to its simplicity constraints, $\operatorname{SEA}_{n, b}$ is based on a limited number of elementary operations (selected for their availability in any processing device) denoted as follows: (1) bitwise $\operatorname{XOR} \oplus$, (2) addition $\bmod 2^{b} \boxplus$, (3) a 3-bit substitution box $S:=\{0,5,6,7,4,3,1,2\}$ that can be applied bitwise to any set of 3-bit words for efficiency purposes. In addition, we use the following rotation operations:

(4) Word rotation $R$, defined on $n_{b}$-word vectors:

$$
\begin{aligned}
R: \mathbb{Z}_{2^{b}}^{n_{b}} \rightarrow \mathbb{Z}_{2^{b}}^{n_{b}}: x \rightarrow y=R(x) \Leftrightarrow \quad & y_{i+1}=x_{i}, 0 \leq i \leq n_{b}-2, \\
& y_{0}=x_{n_{b}-1}
\end{aligned}
$$




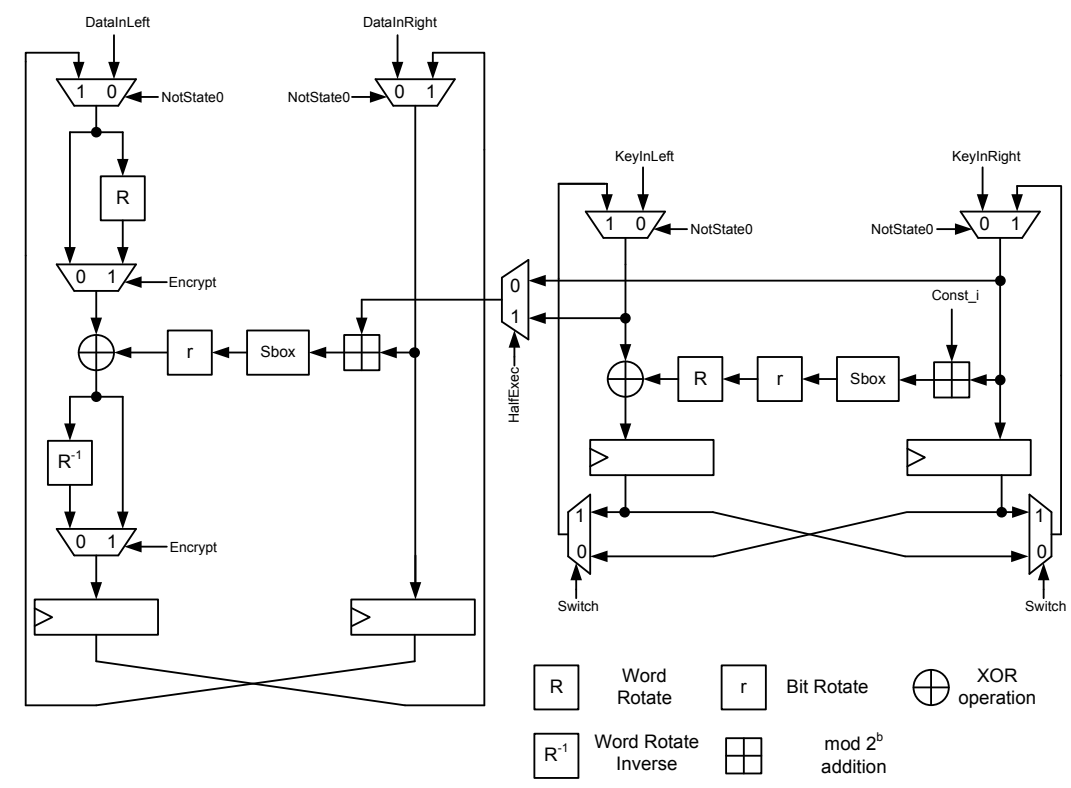

Fig. 2. Loop implementation of SEA.

(5) Bit rotation $r$, defined on $n_{b}$-word vectors:

$$
r: \mathbb{Z}_{2^{b}}^{n_{b}} \rightarrow \mathbb{Z}_{2^{b}}^{n_{b}}: x \rightarrow y=r(x) \Leftrightarrow \quad \begin{aligned}
& y_{3 i}=x_{3 i} \ggg 1, \\
& \\
& y_{3 i+1}=x_{3 i+1}, \\
& y_{3 i+2}=x_{3 i+2} \lll 1,
\end{aligned}
$$

where $0 \leq i \leq \frac{n_{b}}{3}-1$ and $\gg$ and $\lll$ respectively represent the cyclic right and left shifts inside a word.

\section{The round and key round}

Based on the previous definitions, the encrypt round $F_{E}$, decrypt round $F_{D}$ and key round $F_{K}$ are pictured in Figure 1 and defined as:

$$
\begin{aligned}
{\left[L_{i+1}, R_{i+1}\right]=F_{E}\left(L_{i}, R_{i}, K_{i}\right) \Leftrightarrow } & R_{i+1}=R\left(L_{i}\right) \oplus r\left(S\left(R_{i} \boxplus K_{i}\right)\right) \\
& L_{i+1}=R_{i} \\
{\left[L_{i+1}, R_{i+1}\right]=F_{D}\left(L_{i}, R_{i}, K_{i}\right) \Leftrightarrow } & R_{i+1}=R^{-1}\left(L_{i} \oplus r\left(S\left(R_{i} \boxplus K_{i}\right)\right)\right) \\
& L_{i+1}=R_{i} \\
{\left[K L_{i+1}, K R_{i+1}\right]=F_{K}\left(K L_{i}, K R_{i}, C_{i}\right) \Leftrightarrow } & K R_{i+1}=K L_{i} \oplus R\left(r\left(S\left(K R_{i} \boxplus C_{i}\right)\right)\right) \\
& K L_{i+1}=K R_{i}
\end{aligned}
$$

\section{The complete cipher}

The cipher iterates an odd number $n_{r}$ of rounds. The following pseudo-C code encrypts a plaintext $P$ under a key $K$ and produces a ciphertext $C . P, C$ and $K$ have a parametric bit size $n$. The operations within the cipher are performed considering parametric $b$-bit words.

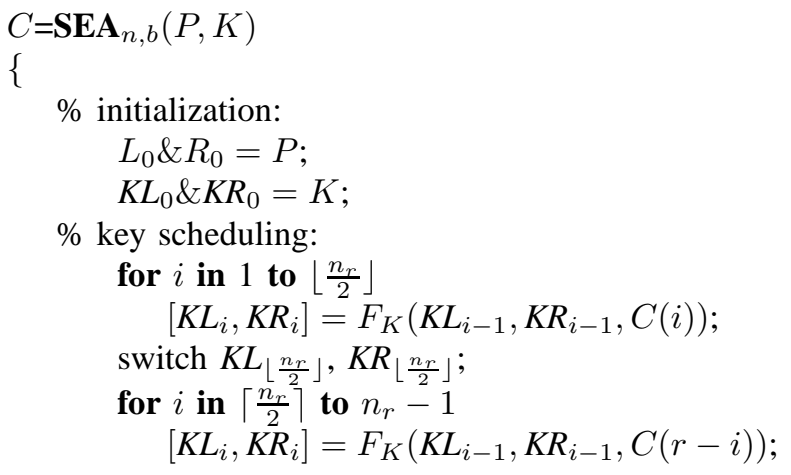

$\%$ encryption:

$$
\begin{aligned}
& \text { for } i \text { in } 1 \text { to }\left\lceil\frac{n_{r}}{2}\right\rceil \\
& \quad\left[L_{i}, R_{i}\right]=F_{E}\left(L_{i-1}, R_{i-1}, K R_{i-1}\right) \\
& \text { for } i \text { in }\left\lceil\frac{n_{r}}{2}\right\rceil+1 \text { to } n_{r} \\
& \quad\left[L_{i}, R_{i}\right]=F_{E}\left(L_{i-1}, R_{i-1}, K L_{i-1}\right) ;
\end{aligned}
$$

$\%$ final:

$C=R_{n_{r}} \& L_{n_{r}}$

switch $K L_{n_{r}-1}, K R_{n_{r}-1}$; \},

where $\&$ is the concatenation operator, $K R_{\left\lfloor\frac{n_{r}}{2}\right\rfloor}$ is taken before the switch and $C(i)$ is a $n_{b}$-word vector of which all the words have value 0 excepted the LSW that equals $i$. Decryption is exactly the same, using the decrypt round $F_{D}$.

\section{IMPLEMENTATION OF A LOOP ARCHITECTURE}

\section{A. Description}

The structure of our loop architecture for SEA is depicted in figure 2, with the round function on the left part and the key schedule on the right part. Resource-consuming blocks are the Sboxes and the $\bmod 2^{b}$ adder; the Word Rotate and Bit Rotate blocks are implemented by swapping wires.

According to the specifications, the key schedule contains two multiplexors allowing to switch the right and left part of the round key at half the execution of the algorithm using the appropriate command signal Switch. The multiplexor controlled by HalfExec provides the round function with the right part of the round key for the first half of the execution and transmits its left part instead after the switch. To support both encryption and decryption, we finally added two multiplexors controlled by the Encrypt signal. Supplementary area consumption will be caused by the two routing pathes. 
TABLE I

IMPLEMENTATION RESULTS FOR SEA WITH DIFFERENT $n$ AND $b$ PARAMETERS

\begin{tabular}{|c|c|c|c|c|c|c|c|c|}
\hline$n$ & $\mathrm{~b}$ & $n_{r}$ & $\begin{array}{c}\sharp \text { of } \\
\text { slices }\end{array}$ & $\begin{array}{c}\sharp \text { of } \\
\text { slice FFs }\end{array}$ & $\begin{array}{c}\text { Output every } \\
\text { cycle }\end{array}$ & $\begin{array}{c}\text { Freq } \\
(\mathrm{MHz})\end{array}$ & $\begin{array}{c}\text { Throughput } \\
\text { (Mbits/sec) }\end{array}$ & $\begin{array}{c}\text { Thr./Area } \\
\text { Mbits/sec /slice }\end{array}$ \\
\hline \hline 48 & 4 & 55 & 197 & 127 & $1 / 55$ & 237 & 207 & 1.049 \\
48 & 8 & 51 & 176 & 131 & $1 / 51$ & 234 & 220 & 1.250 \\
\hline 72 & 4 & 77 & 296 & 194 & $1 / 77$ & 243 & 228 & 0.769 \\
72 & 6 & 73 & 258 & 194 & $1 / 73$ & 242 & 238 & 0.924 \\
72 & 12 & 73 & 263 & 198 & $1 / 73$ & 242 & 239 & 0.908 \\
\hline 96 & 4 & 95 & 368 & 241 & $1 / 95$ & 242 & 244 & 0.663 \\
96 & 8 & 93 & 333 & 246 & $1 / 93$ & 238 & 245 & 0.737 \\
\hline 108 & 6 & 111 & 376 & 280 & $1 / 111$ & 241 & 235 & 0.625 \\
\hline 126 & 7 & 117 & 438 & 328 & $1 / 117$ & 241 & 260 & 0.593 \\
\hline 132 & 11 & 121 & 448 & 330 & $1 / 121$ & 227 & 248 & 0.554 \\
\hline 144 & 4 & 149 & 604 & 376 & $1 / 149$ & 241 & 233 & 0.385 \\
144 & 6 & 139 & 488 & 359 & $1 / 139$ & 241 & 250 & 0.512 \\
144 & 8 & 135 & 496 & 371 & $1 / 135$ & 241 & 257 & 0.518 \\
144 & 12 & 133 & 478 & 352 & $1 / 133$ & 223 & 236 & 0.495 \\
\hline
\end{tabular}

The algorithm can easily beneficiate of a modular implementation, taking as only mandatory parameters the size of the plaintexts and keys $n$ and the word length $b$. The number of rounds $n_{r}$ is an optional input that can be automatically derived from $n$ and $b$ according to the guidelines given in [1]. From the datapath description of Figure 2, a scalable design can then be straightforwardly obtained by using generic VHDL coding. A particular care only has to be devoted to an efficient use of the mod $2^{b}$ adders in the key scheduling part.

In the round function, the mod $2^{b}$ adders are realized by using $n_{b} b$-bits adders working in parallel without carry propagation between them. However, in the key schedule, the signal Const_i (provided by the control part) can only take a value between 0 and $\frac{n_{r}}{2}$. Therefore, it may not be necessary to use $n_{b}$ adders. If $\log _{2}\left(\frac{n_{r}}{2}\right) \leq b$, then a single adder is sufficient. If $\log _{2}\left(\frac{n_{r}}{2}\right)>b$, then $\left\lceil\frac{\log _{2}\left(\frac{n_{r}}{2}\right)}{b}\right\rceil$ adders will be required. In the next section, we detail the implementation results of this architecture for different parameters.

\section{B. Implementation results}

Implementation results were extracted after place and route with the ISE 7.1i tool from Xilinx on a xc4vlx25 VIRTEX4 platform with speed grade -12 . In order to illustrate the modularity of our architecture, we ran the design tool for different sets of parameters, with plaintext/key sizes $n$ ranging from 48 to 144 bits and word lengths of $4,6,7,8$, and 12 bits. For the control part, we used the recommended number of rounds $n_{r}=\left[3 \frac{n}{4}+2\left(\frac{n}{2 b}+\frac{b}{2}\right)\right]^{1}$. The computed implementation costs stand for both the operative and control parts.

A summary of these results is presented in table I, where the area requirements (in slices), the work frequency and the throughput are provided. We observe that the obtained values for the work frequency are very close for all the implementations. Indeed, the critical path (passing through the key scheduling multiplexors, a mod $2^{b}$ adder, the Round Function Sbox, a XOR operator and the multiplexor selecting between encryption or decryption pathes) is very similar for any of our selected values for $n$ and $b$.

\footnotetext{
${ }^{1}+1$ if this term is even.
}

For a given $n$ value, it is noticeable that increasing $b$ decreases the number of rounds $n_{r}$ and therefore improves the throughput (since work frequencies are close in all our examples). Similarly, for our set of parameters, increasing $b$ for a given $n$ generally decreases the area requirements in slices. These observations lead to the empirical conclusion that, as long as the b parameter is not a limiting factor for the work frequency, increasing the word size leads to the most efficient implementations for both area and throughput reasons.

\section{Comparisons with other block ciphers}

For our comparative discussions, we reported a few implementation results of the AES Rijndael in Table II. We selected the implementations in [7], [8] and [9] because their design choices fit relatively well with those of the presented SEA architectures. Mainly, these cores do not take advantage of RAM blocks nor loop unrolling. The four first cores all correspond to loop architectures with a 128-bit datapath. They respectively have no pipeline (Pipe0) or a 3-stage pipeline (Pipe3) and use LUT-based or distributed RAM-based Sboxes. The fifth referenced implementation [7] uses a 32-bit datapath and consequently reduces the area requirements at the cost of a smaller throughput. Finally, [8] uses a 128-bit datapath with a pipelined composite field description of the Sbox. As a matter of fact, a lot of other FPGA implementations of the AES can be found in the open literature, e.g. taking advantage of different datapath sizes, FPGA RAM blocks, pipelining, unrolling techniques, ..., e.g. [10], [11], [12] and [13].

Additionally, we compared these results with those obtained for ICEBERG, a block cipher optimized for reconfigurable hardware devices. Details on the ICEBERG architecture and different possible implementation tradeoffs are discussed in [14]. The reported result corresponds to a single-round loop architecture without pipeline. Compared to the AES Rijndael, ICEBERG is built upon a combination of 4-bit operations that perfectly fit into the FPGAs LUTs which intently results in a very good ratio between throughput and area.

The implementation results in Table II lead to the following observations. First, in terms of area requirements (for a datapath size equal to the block size), SEA generally exhibits the 
TABLE II

IMPLEMENTATION RESULTS OF OTHER BLOCK CIPHERS.

\begin{tabular}{|c|c|c|c|c|c|c|c|c|}
\hline Algorithm & Device & $n_{r}$ & $\mathrm{E} / \mathrm{D}$ & $\begin{array}{l}\sharp \text { of } \\
\text { slices }\end{array}$ & $\begin{array}{c}\text { Freq } \\
(\mathrm{MHz})\end{array}$ & $\begin{array}{l}\text { Throughput } \\
\text { (Mbits/sec) }\end{array}$ & $\begin{array}{c}\text { Thr./Area } \\
\text { Mbits/sec /slice }\end{array}$ & $\mathrm{bit} / \mathrm{slice}$ \\
\hline AES (Pipe0-LUT) [9] & $\mathrm{xc} 2 \mathrm{v} 400$ & 10 & no & 2744 & 59 & 760 & 0.277 & 0.047 \\
\hline AES (Pipe0-Dist) [9] & $\mathrm{xc} 2 \mathrm{v} 400$ & 10 & no & 1780 & 78 & 1000 & 0.562 & 0.072 \\
\hline AES (Pipe3-LUT) [9] & $\mathrm{xc} 2 \mathrm{v} 400$ & 10 & no & 2909 & 148 & 1890 & 0.650 & 0.044 \\
\hline AES (Pipe3-Dist) [9] & $\mathrm{xc} 2 \mathrm{v} 400$ & 10 & no & 1940 & 178 & 2280 & 1.175 & 0.066 \\
\hline AES [7] & xcv100e & 10 & yes & 1125 & 161 & 215 & 0.191 & 0.114 \\
\hline AES [8] & xcv3200e & 10 & no & 1769 & 167 & 2085 & 1.179 & 0.072 \\
\hline ICEBERG & $\mathrm{xc} 4 \mathrm{vlx} 25$ & 16 & yes & 575 & 247 & 988 & 1.718 & 0.111 \\
\hline $\mathrm{SEA}_{126,7}$ & xcv3200e & 117 & yes & 434 & 92 & 99 & 0.228 & 0.290 \\
\hline $\mathrm{SEA}_{126,7}$ & $\mathrm{xc} 2 \mathrm{v} 4000$ & 117 & yes & 424 & 145 & 156 & 0.368 & 0.302 \\
\hline SEA $_{126,7}$ & $\mathrm{xc} 4 \mathrm{v} 1 \times 25$ & 117 & yes & 438 & 241 & 260 & 0.594 & 0.288 \\
\hline
\end{tabular}

smallest cost. Measuring the area efficiency with the bit per slice metric leads to a similar conclusion. Of course, the area requirements of, e.g. the AES Rijndael could still be decreased by using smaller datapaths [15] and such a comparative table only serves as an indicator rather than a strict comparison. However, in the present case, these results clearly suggest the low-cost purpose of our presented implementations.

By contrast, looking at the throughput per area metric indicates that these low area requirements come with weak throughputs. This is of course mainly due to the high number of rounds in SEA. With this respect, it is interesting to compare SEA and ICEBERG since their implementation results clearly illustrate their respective context/platform-oriented design approach. Namely SEA is purposed for low cost applications while ICEBERG optimizes the throughput per slice.

These numbers also confirm the differences between specialized algorithms and standard solutions. It must be underlined with this respect that the AES Rijndael still ranges relatively well in terms of hardware cost and throughput efficiency, compared to the investigated specialized solutions. Note also that SEA was initially purposed for low cost software implementations. While these design criteria turned out to allow low cost hardware implementations as well, it is likely that targeting a cipher specifically for low cost hardware would lead to even better solutions, e.g. [16].

Finally, it is also important to emphasize a number of advantages in SEA that cannot be found in other recent block ciphers, namely its simplicity, scalability (re-implementing SEA for a new block size does not require to re-write code), good combination of encryption and decryption and ability to derive keys "on the fly" both in encryption and decryption.

\section{CONCLUSION}

This letter presented FPGA implementations of a scalable encryption algorithm for various sets of parameters. The presented parametric architecture allows keeping the flexibility of the algorithm by taking advantage of generic VHDL coding. It executes one round per clock cycle, computes the round and the key round in parallel and supports both encryption and decryption at a minimal cost. Compared to other recent block ciphers, SEA exhibits a very small area utilization that comes at the cost of a reduced throughput. Consequently, it can be considered as an interesting alternative for constrained environments. Scopes for further research include low power ASIC implementations purposed for RFIDs as well as further cryptanalysis efforts and security evaluations.

\section{REFERENCES}

[1] F.-X. Standaert, G. Piret, N. Gershenfeld, and J.-J. Quisquater, "SEA: A Scalable Encryption Algorithm for Small Embedded Applications," in the Proceedings of CARDIS 2006, ser. LNCS, vol. 3928, Taragona, Spain, 2006, pp. 222-236.

[2] Data Encryption Standard, NIST Federal Information Processing Standard FIPS 46-1, Jan. 1998.

[3] J. Daemen, V. Rijmen, The Design of Rijndael. Springer-Verlag, 2001.

[4] Advanced Encryption Standard, NIST Federal Information Processing Standard FIPS 197, Nov. 2001.

[5] D. Wheeler and R. Needham, "TEA, a Tiny Encryption Algorithm," in the Proceedings of Fast Software Encryption - FSE 1994, ser. LNCS, vol. 1008, Leuven, Belgium, Dec. 1994, pp. 363-366.

[6] G. Yuval, "Reinventing the Travois: Encryption/MAC in 30 ROM Bytes," in the Proceedings of Fast Software Encryption - FSE 1997, ser. LNCS, vol. 1267, Haifa, Israel, Jan. 1997, pp. 205-209.

[7] N. Pramstaller and J. Wolkerstorfer, "A Universal and Efficient AES Coprocessor for Field Programmable Logic Arrays," in the Proceedings of FPL 2004, LNCS, vol. 3203, Leuven, Belgium, Aug. 2004, pp. 565-574.

[8] F.-X. Standaert, G. Rouvroy, J.-J. Quisquater, and J.-D. Legat, "Efficient Implementation of Rijndael Encryption in Reconfigurable Hardware: Improvements and Design Tradeoffs," in the Proceedings of Cryptographic Hardware and Embedded Devices - CHES 2003, ser. LNCS, vol. 2779, Cologne, Germany, Sep. 2003, pp. 334-350.

[9] J. Zambreno, D. Nguyen, and A. Choudhary, "Exploring Area/Delay Tradeoffs in an AES FPGA implementation," in the Proceedings of FPL 2004, ser. LNCS, vol. 3203, Leuven, Belgium, Aug. 2004, pp. 575-585.

[10] K. Gaj and P. Chodowiec, "Fast Implementation and Fair Comparison of the Final Candidates for Advanced Encryption Standard Using Field Programmable Gate Arrays," in Topics in Cryptology - CT-RSA 2001, LNCS., vol. 2020, San Fransisco, USA, pp. 84-99.

[11] G. P. Saggese, A. Mazzeo, N. Mazzocca, and A. G. M. Strollo, "An FPGA-Based Performance Analysis of the Unrolling, Tiling, and Pipelining of the AES Algorithm," in the Proceedings of FPL 2003, ser. LNCS, vol. 2778, Lisbon, Portugal, Sep. 2003, pp. 292-302.

[12] A. J. Elbirt, W. Yip, B. Chetwynd, and C. Paar, "An FPGA Implementation and Performance Evaluation of the AES Block Cipher Candidate Algorithm Finalists," in AES Candidate Conference, 2000, pp. 13-27.

[13] K. Jarvinen, M. Tommiska, J. Skytta, "Comparative Survey of HighPerformance Cryptographic Algorithm Implementations on FPGAs," IEE Proceedings on Information Security, vol. 152, Oct. 2005, pp. 3-12.

[14] F.-X. Standaert, G. Piret, G. Rouvroy, and J.-J. Quisquater, "FPGA Implementations of the ICEBERG Block Cipher," in the Proceedings of ITCC 2005, vol. 1, Las Vegas, USA, Apr. 2005, pp. 556-561.

[15] M. Feldhofer, J. Wolkerstorfer, and V. Rijmen, "AES Implementation on a Grain of Sand," in IEE Proceedings on Information Security, vol. 152. IEE, Oct. 2005, pp. 13-20.

[16] D. Hong, J. Sung, S. Hong, J. Lim, S. Lee, B.-S. Koo, C. Lee, D. Chang, J. Lee, K. Jeong, J. Kim, and S. Chee, "HIGHT: a New Block Cipher Suitable for Low-Resource Devices," in The Proceedings of Cryptographic Hardware and Embedded Devices - CHES 2006, ser. LNCS, vol. 4249, Yokohama, Japan, Oct. 2006, pp. 13-20. 